\title{
Estrategia depromoción de valores a través del fútbol en la escuela «Frida Kahlo» de la ciudad Strategy to promote values through school football «Frida Kahlo» of the city
}

\author{
Jesús Jasso Reyes, Carlos Raúl Almanza Rodríguez, Miguel Conchas Ramírez, Javier Rivero Carrasco, Roberto \\ Paredes Carrera, Daniel Jaime Jiménez Mireles \\ UniversidadAutónoma de Chihuahua (México)
}

\begin{abstract}
Resumen: La investigación «Estrategias de Promoción de Valores a través del Futbol», se realizó en la Escuela Primaria Frida Kahlo de la ciudad de Chihuahua Chih. México, sobre la pregunta: ¿Hasta qué punto se pueden promover los valores mediante una estrategia didáctica que tenga como eje central la práctica del futbol? El objetivo general es impulsar la formación de los valores a través de la educación deportiva, con la participación de actores importantes como: padres de familia, maestros y alumnos. Los valores que se desarrollaron en los alumnos fueron: responsabilidad, respeto, disciplina, trabajo en equipo y compañerismo. Diseñándose y aplicándose un proyecto de intervención educativa para alumnos de $4^{\circ}$ y $5^{\circ}$ grado, 22 niños y 8 niñas siendo un total de 30, generando cambios significativos y reconociendo que a través de la educación deportiva se pueden realizar cambios actitudinales y de valores en los alumnos mismos.
\end{abstract}

Palabras clave: valores; deporte; estrategia.

Abstract: The research «Strategies of promotion values through football», was conducted at the Frida Kahlo primary school in the city of Chihuahua, Chih. Mexico, on the question: to what extent can promote values through a didactic strategy that has as its central focus the practice of football? The overall objective is to encourage the formation of values through sports education, with the participation of important actors as: parents, teachers and students. The values that were developed in the students were: responsibility, respect, discipline, teamwork and camaraderie. Designing and implementing a project of educational intervention for students of 4th and 5th grade, 22 and 8 children, being a total of 30, generating significant changes and recognizing that attitudinal changes be made through sport education and values in the same students.

Key words: values; sport; strategy.

\section{Introducción}

La sociedad actual reclama con insistencia una educación en valores para niños, adolescentes y jóvenes. Es imprescindible afrontar la plaga de analfabetismo moral que se va extendiendo en las capas más jóvenes e indefensas de la sociedad.A raíz de esto se puede observar en la escuela la falta de valores en los alumnos desde los de primer grado hasta los de sexto grado, en los pasillos, áreas de juego, explanada y los propios salones, donde el maestro en muchas ocasiones es rebasado por el alumno y se ve obligado a utilizar métodos más fuertes para lograr controlar la disciplina en el alumno.

La investigación «Estrategias de Promoción de Valores a través del Fútbol», se realizó en la Escuela Primaria Frida Kahlo de la ciudad de Chihuahua Chih. México, partiendo de la pregunta: ¿Hasta qué punto se pueden promover los valores de respeto, la responsabilidad, el trabajo en equipo, el compañerismo, etc. mediante una estrategia didáctica que tenga como eje central la práctica del fútbol?, cuyo objetivo general es impulsar la formación de los valores a través de la educación deportiva, con la participación de actores importantes para su fomento y desarrollo, como: padres de familia, maestros y alumnos. Los valores que se desarrollaron en los alumnos fueron: responsabilidad, respeto, disciplina, trabajo en equipo y compañerismo.

\section{Materiales y métodos}

El término intervención no es unívoco. Es más bien ambiguo, multifacético, camaleónico... Así, puede denotar; corrección, educación, enriquecimiento, prevención, rehabilitación, modificación, remedio, prestación de servicios, estimulación, mejoría, terapia, entrenamiento, tratamiento..., y hasta la no intervención puede considerarse una forma de intervención (Román \& García, 1990). Para efectos de este estudio seguimos la secuencia metodológica sugerida por la mayoría de los autores, a partir de los siguientes elementos:

Fecha recepción: 01-07-12- Fecha envío revisores: 01-07-12- Fecha de aceptación: 14-12-12 Correspondencia: Jesús Jasso Reyes

E-mail; jjasso@uach.mx

\section{Diagnóstico de necesidades}

La sociedad actual reclama con insistencia una educación en valores para niños, adolescentes y jóvenes. Es imprescindible afrontar la plaga de analfabetismo moral que se va extendiendo en las capas más jóvenes e indefensas de la sociedad. Araíz de esto se puede observar en la escuela la falta de valores en los alumnos desde los de primer grado hasta los de sexto grado, en los pasillos, áreas de juego, explanada y los propios salones, donde el maestro en muchas ocasiones es rebasado por el alumno y se ve obligado a utilizar métodos más fuertes para lograr controlar la disciplina en el alumno.

Es por esto que se presenta este proyecto con la finalidad de disminuir y erradicar este tipo de malos hábitos a través de la práctica del fútbol utilizando diferentes actividades así como la colaboración del personal docente y los padres de familia para el logro del objetivo general.

\section{Objetivo de la intervención}

Impulsar la formación de valores desde la práctica de los mismos en los espacios escolares de la primaria Frida Kahlo, a través del deporte, concretamente del fútbol.

\section{Contenido de la intervención}

Se promoverán en la práctica del fútbol los valores de Trabajo en Equipo, del Respeto, Responsabilidad, Disciplina, y Compañerismo.

\section{Contexto de desarrollo}

Contexto natural: Este proyecto se realizó en la Escuela Primaria Federal Frida Kahlo T. M. en un espacio adecuado para utilizarse como campo de fútbol.

\section{Destinatarios de la intervención}

Participaron en este proyecto los alumnos de $4^{\circ}$ y $5^{\circ}$ grado con edades aproximadas entre 9 y 11 años de edad. Se trabajara con un equipo de fútbol en el cual 22 son niños y 8 niñas, dando un total de 30 alumnos.

\section{Contenido de la estrategia}

Promoción de valores a través del fútbol en la escuela «Frida Kahlo» de la ciudad de Chihuahua, Chih. México. 


\section{Resultados}

Una vez que se procesó, analizó y discutió la información de base surgida de la intervención llevada a cabo, se pudo identificar una serie de cambios en el aspecto actitudinal y de tipo axiológico que nos permite aseverar que sí es, precisamente el deporte y su práctica, orientada hacia la promoción de valores, un instrumento valiosísimo para alcanzar ese propósito. Veamos tan solo los resultados que consideramos más representativos en el estudio realizado.

\section{Valor «Trabajo en Equipo»}

Los datos de los alumnos que no asumieron su rol dentro del proyecto son mínimos y aun así disminuye el número en su fase final resaltando la diferencia del $10 \%$.

Los alumnos que no asumieron su rol dentro del proyecto son mínimos y aun así disminuye el número en su fase final resaltando la diferencia del $10 \%$

La mayoría de los alumnos se identificaron con el proyecto en su etapa inicial, resaltando una diferencia para la etapa final de $16.6 \%$ dando como resultado que solamente un alumno fue el que no se identificó.

\section{Valor «Respeto»}

Una vez terminada la intervención la asistencia aumento un 19.2\%, en faltas la disminución es de 15\% y de retardos disminuye el 3.4\%

Se denota con claridad que el valor de puntualidad aumento en un 5.7\% atribuible al impacto del programa aplicado.

Respecto al vocabulario: en la fase inicial los alumnos sin problema fue el $63.3 \%$ y en la fase final el $76.6 \%$ estableciendo una diferencia de 13.3\% en el mejoramiento de este problema.

\section{Valor «Responsabilidad»}

De un 20.8\% de ellos no lograban el propósito del proyecto, a partir de la fase media lograron asumir su responsabilidad al $100 \%$.

\section{Valor «Disciplina»}

Los alumnos con este problema fue de un $10 \%$ disminuyendo a un 3.3\% logrado en base al impacto del proyecto en ellos, una diferencia de $6.6 \%$ en disminución al problema.

El número de alumnos que protestaban disminuye en la parte final en un $30 \%$ ya que el alumno termina por entender el objetivo del proyecto.

\section{Valor «Compañerismo»}

El número de alumnos que presentaron este problema fue mínimo, pero aun asíse establece una diferencia de 6.6\% en mejora a esteproblema.

La comparación entre la fase inicial y la fase final es la disminución de este problema en un $10 \%$ resaltando que los alumnos aceptaron a sus compañeros con sus diferentes capacidades y deficiencias.

\section{Discusión}

Al término de la intervención de la estrategia para la promoción de valores a través del futbol se pudo detectar que en relación a los valoresobjetivos, la asistencia aumento un $19.2 \%$, en faltas la disminución es de $15 \%$ y de retardos disminuye el $3.4 \%$. Se denota con claridad que el valor de puntualidad aumento en un 5.7\% atribuible al impacto del programa aplicado.

Respecto al vocabulario: en la fase inicial los alumnos sin problema fue el $63.3 \%$ y en la fase final el $76.6 \%$ estableciendo una diferencia de 13.3\% en el mejoramiento del uso adecuado del lenguaje.

El número de alumnos que protestaban disminuye en la parte final en un $30 \%$ ya que el alumno termina por entender el objetivo del proyecto. La comparación entre la fase inicial y la fase final es la disminución de este problema en un 10\% resaltando que los alumnos aceptaron a sus compañeros con sus diferentes capacidades y deficiencias.

\section{Conclusiones}

Se considera que este proyecto deja una profunda huella en todos los alumnos involucrados en el mismo, ya que los resultados que arrojaron causaron un impacto positivo en los alumnos, maestros y padres de familia, dándose cambios actitudinales en ellos dentro y fuera de la escuela. Destacan el valor del respeto y la responsabilidad que causaron gran impacto, así como el del compañerismo, dadas las condiciones del entorno socioeconómico de la escuela.

Se puede asegurar que las estrategias utilizadas en ese proyecto fueron adecuadas, si se considera que la mayoría de ellas fueron las que lograron un impacto positivo en los alumnos, ya que como se demostró en los resultados, los cambios conductuales en los alumnos conforme avanzaba el proyecto, fueron de consideración y que estos llevaron al éxito del proyecto.

Confirmamos que es viable y adecuado aplicar este tipo de proyectos en otros niveles educativos ya que la necesidad de fomentar los valores en los niños y jóvenes es de gran importancia, y que mejor que hacerlo a través del deporte cuya actividad es del agrado de la gran mayoría de ellos.

\section{Referencias}

Bourdieu, P. (1997). Capital cultural, escuela y espacio social. Editorial siglo XXI, México.

Baudrillard, J. (2005). The consumer society: myths and Structure. Sage publications. USA.

Calero (2002). Educación en valores. Editorial San Marcos, Lima. Perú.

Cortez, J., \& Martínez, A. (1996). Diccionario de Filosofía. Editorial Heder S.A. Barcelona, España.

Colectivo de autores (1998). Congreso Comunidad 98, IMDEC. La Habana, Cuba.

Delors, J.(1999). La educación encierraun tesoro, informe a la UNESCO de la comunicación de la comisión internacional sobre la educación para el SIGLO XXI.

Debord, G. (1997). Society of the Spectacle 1967, Translation: Black and Red, HTML Markup: Grez Adargo, USA.

Denis, L. (2000). Ética y Docencia, fondo editorial de la Universidad Pedagógica experimental Libertador. Caracas, Venezuela.

González P. (1994). Currículo: Diseño, práctica y evaluación, CEPES. La Habana, Cuba.

González, V.(2001). LaEducación en valores en el currículo universitario. Disponible en http://www.campus-oei.org/valores/maura.htp

Keel, R. (2005). The McDonalization of Society, Introduction to sociology. Disponible en http://www.umsl.edu/-rkeel/010/ mcdonsoc.html.

Moreno, M. (1999), Valores transversales en el currículo. Revista de Educación y Cultura, sección 47. México.

Santos, M. A., (2002). Currículo oculto y aprendizaje en valores. INET. Tema 21. Málaga, España.

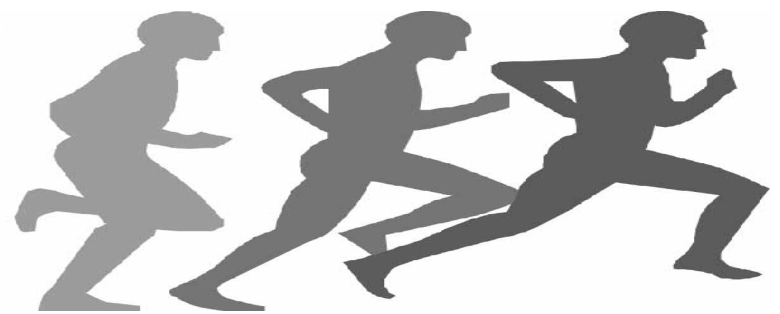

